\title{
Dirac Leptogenesis in assistance of Dark Matter and Neutrino Mass
}

\author{
Nimmala Narendra* \\ Department of Physics, Indian Institute of Technology, Hyderabad, Kandi, Sangareddy, 502285, \\ Telangana, India \\ E-mail: ph14resch01002@ith.ac.in
}

\section{Nirakar Sahoo}

Department of Physics, Indian Institute of Technology, Hyderabad, Kandi, Sangareddy, 502285,

Telangana, India

E-mail: nirakar.pintu.sahoo@gmail.com

\section{Narendra Sahu}

Department of Physics, Indian Institute of Technology, Hyderabad, Kandi, Sangareddy, 502285,

Telangana, India

E-mail: nsahueith.ac.in

\begin{abstract}
We propose an extension of the standard model with $U(1)_{D} \times U(1)_{B-L} \times Z_{2}$ symmetry. With this extension of the model we assumed that the neutrinos are Dirac (i.e. $B-L$ is an exact symmetry), and found a simultaneous solution for non zero neutrino masses, Dark Matter(DM) content and the baryon asymmetry of the Universe. The observed baryon assymmetry of the Universe explained through the Dirac leptogenesis with the assistance of DM. The $U(1)_{D}$ symmetry is broken at a $\mathrm{TeV}$ scale and gives mass to a neutral gauge boson $Z_{D}$. At one loop level this neutral gauge boson mixes with the standard model Z-boson and paves a path to detect the DM at terrestrial laboratories through spin independent elastic scattering. We explain the neutrino mass through a Lagrangian term, which softly broken by the $Z_{2}$ symmetry.
\end{abstract}

ICHEP 2018, 39th International Conference on High Energy Physics

4-11 July 2018

Seoul, South Korea

${ }^{*}$ Speaker. 


\section{Introduction}

Till now there is no evidence found in favour to Majorana nature of neutrinos from neutrinoless double beta decay experiments [1]. So still there is a possibility that the neutrinos might be Dirac in nature. Even though neutrinos are Dirac in nature (i.e. $B-L$ is exactly conserved), the Baryon Asymmetry of the Universe(BAU) can be explained through Dirac leptogenesis [2, 3], which connects the Dirac neutrino mass with the observed BAU. This mechanism works on the point that the equilibration time between left and right-handed neutrinos mediated through SM Higgs (i.e. $\left.Y \overline{v_{R}} H v_{L}\right)$ is much less than $(B+L)$ violating sphaleron transitions above electroweak phase transition. If we can write that $B-L=B-\left(L_{\mathrm{SM}}+L_{v_{R}}\right)=0$ [4], then we see that a net $B-L_{\mathrm{SM}}$ is generated in terms of $L_{v_{R}}$. The electroweak sphalerons will not act on $L_{v_{R}}$, while the non-zero $B-L_{\mathrm{SM}}$ will be converted to a net baryon asymmetry via $B+L$ violating sphaleron transitions.

\section{The model and Dirac mass of neutrinos}

We extended the SM model with additonal symmetries $U(1)_{B-L} \times U(1)_{D} \times Z_{2}$ and the particle content with three right handed neutrinos $v_{R}$ which play a role in anomaly cancellation and they are odd under $Z_{2}$, with lepton number as 1 . We also introduced $\chi$ and $\psi$ vector-like fermions which are singlet and doublet under $S U(2)_{L}$ respectively. They are odd under $Z_{2}$ with $U(1)_{D}$ charge as 1 and the lepton number is same as $v_{R}$. And two heavy scalars $X_{1,2}$, which are odd under $Z_{2}$, we introduce here creates equal and opposite asymmetry in both $\ell$ (left sector) and $v_{R}$ (right sector) in the visible sector and also creates an asymmetry in both $\chi$ and $\psi$ in dark sector, in their CP-violating out-of-equilibrium decay. The lepton asymmetry stored in the left sector converts to a net baryon asymmetry through $B+L$ violating sphaleron transitions. And the asymmetry which is stored in the right sector remains uneffected until the temperature much below the electroweak phase transion. The asymmetry in $\psi$ converts to $\chi$ asymmetry in its out-of-euilibrium decays and we can show that its contribution to the relic abundance is negligible. The lightest particle $\chi$ amongst the dark sector paricles acts as DM candidate. In this model the $U(1)_{D}$ is broken symmetry and gives mass to the gauge boson $Z_{D}$, while the $U(1)_{B-L}$ is not a broken symmetry and guarantees the Dirac nature of the neutrinos. The relevant Lagrangian can be written as:

$$
\mathscr{L} \supset M_{\psi} \bar{\psi} \psi+M_{\chi} \bar{\chi} \chi+\left[f_{k l} \overline{\ell_{k}} \tilde{X} v_{R l}+\lambda \bar{\psi} \tilde{X} \chi+\text { h.c. }\right]-V(H, X),
$$

where

$$
V(H, X)=-M_{H}^{2} H^{\dagger} H+M_{X}^{2} X^{\dagger} X+\lambda_{H}\left(H^{\dagger} H\right)^{2}+\lambda_{X}\left(X^{\dagger} X\right)^{2}+\lambda_{H X}\left(H^{\dagger} H\right)\left(X^{\dagger} X\right) .
$$

We allow the $Z_{2}$ symmetry to break softly[5] via:

$$
\mathscr{L}_{\text {soft }}=-\mu^{2} H^{\dagger} X+\text { h.c. }
$$

As a result the Dirac mass of the neutrinos can be generated at tree level. After integrating out the tree level diagram we get the Dirac neutrino mass:

$$
M_{v}=\frac{f\langle H\rangle \mu^{2}}{M_{X}^{2}}
$$

where $\langle H\rangle=174 \mathrm{GeV}$, is the Higgs vacuum expectation value. To generate required Dirac masses of the neutrinos of order $0.1 \mathrm{eV}$, we need the ratio of $\frac{\mu}{M_{X}} \approx 10^{-4}$, assuming here $f \sim 10^{-4}$. 


\section{Lepton asymmetry and dark matter abundance from X-decay}

The presence of X-particles and their interactions the mass matrix of X can be replaced with new mass matrix and after diagonalising the new mass matrix we get new mass eigenstates $\xi_{1}^{ \pm}$and $\xi_{2}^{ \pm}$. For further details [6]. The CP-violation arises via the interference of tree level and one loop self energy correction diagrams and the asymmetry $\varepsilon_{L}$ in the visible sector can be calculated. Using the CP-asymmetry $\varepsilon_{L}$, we can estimate the generated lepton asymmetry $Y_{L} \equiv \frac{n_{L}}{s}$. The abundance of $\xi$ and the amount of lepton asymmetry can be calculated using Boltzmann equations as,

$$
\frac{d Y_{\xi_{1}}}{d x}=-\frac{x}{H\left(M_{\xi_{1}}\right)} s<\sigma|v|_{\left(\xi_{1} \xi_{1} \rightarrow A l l\right)}>\left[Y_{\xi_{1}}^{2}-Y_{\xi_{1}}^{e q 2}\right]-\frac{x}{H\left(M_{\xi_{1}}\right)} \Gamma_{\left(\xi_{1} \rightarrow A l l\right)}\left[Y_{\xi_{1}}-Y_{\xi_{1}}^{e q}\right]
$$

and

$$
\frac{d Y_{L}}{d x}=\varepsilon_{L} \frac{x}{H\left(M_{\xi_{1}}\right)} \Gamma_{\left(\xi_{1} \rightarrow A l l\right)} B_{L}\left[Y_{\xi_{1}}-Y_{\xi_{1}}^{e q}\right]
$$

where the $x=\frac{M_{\xi_{1}}}{T}$, is the dimensionless variable. The number density of $\xi$ and the lepton asymmetry are shown in Fig. 1(left panel). Here we have taken coupling constant $\mathrm{f}$ as $10^{-4}$ and the $\lambda$ as $0.47 \times 10^{-7}$ and the typical cross-section $\sigma|v|_{\xi_{1}} \xi_{1} \rightarrow A l l=10^{-25} \mathrm{GeV}^{-2}$. The branching ratio is of the $\mathscr{O}(1)$ and $M_{\xi_{1}}=10^{10} \mathrm{GeV}$. The sphalerons can transfer a partial lepton asymmetry to a net baryon asymmetry as $Y_{B}=-0.55 Y_{L}$. For $\varepsilon_{L}=2 \times 10^{-7}$ and with the parameters we discussed above gives the baryon asymmetry, $Y_{B}=-1 \times 10^{-10}$.

The relic abundance of $\chi$ can come from the CP-conserving and CP-non-conserving decay of heavy scalar $\xi$, which is nothing but sum of symmetric and asymmetric components of $\chi$, respectively. However we get the contribution of the abundance of the asymmetric component of DM to the correct relic abundance is very small, due to small CP-asymmetry and small branching ratio $B_{\chi} \equiv \operatorname{Br}\left(\xi_{1} \rightarrow \psi \chi\right)$, therefore we neglect it. The symmetric component of $\chi$ from the decay of $\xi_{1}$ and $\xi_{2}$ can be estimated from Boltzmann equations as,

$$
\frac{d Y_{\chi}}{d x}=\frac{x}{H\left(M_{\xi_{1}}\right)} \Gamma_{\left(\xi_{1} \rightarrow A l l\right)} B_{\chi}\left[Y_{\xi_{1}}-Y_{\xi_{1}}^{e q}\right]
$$

where $Y_{\xi_{1}}$ given in Eq.3.1. The solutions of Eqs. 3.1 and 3.3 are shown in Fig. 1(right panel).
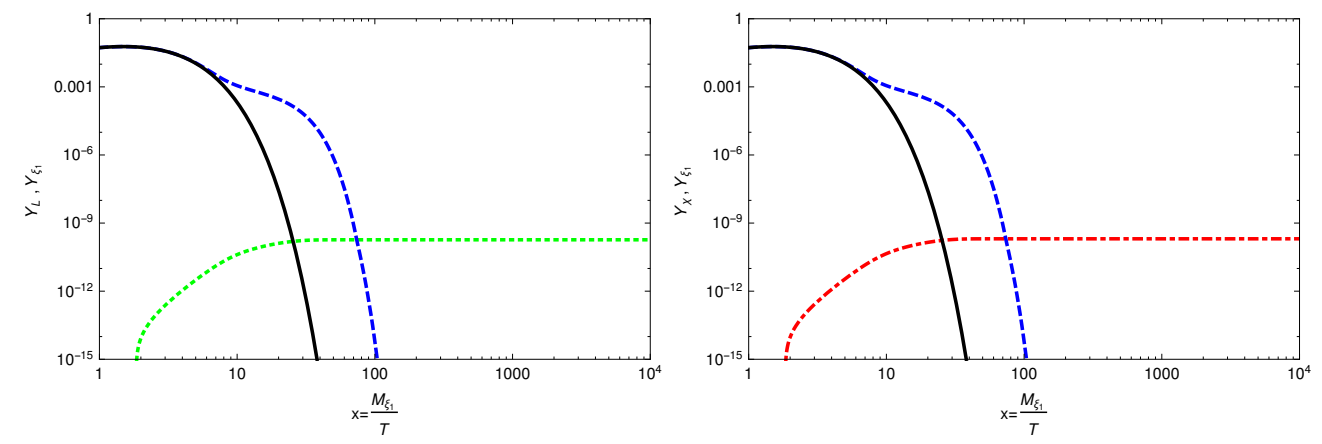

Figure 1: In the left panel the generated lepton asymmetry from the decay of $\xi_{1}$. The Green dotted line shows the abundance of lepton asymmetry. In the right panel the Red dotted line shows the abundance of $\chi \mathrm{DM}$. The Blue dashedline shows the abundance of $\xi_{1}$ and the Black solid-line shows the equilibrium number density of $\xi_{1}$. 
Here we have taken the same parameter space as we discussed in the above paragraph and for the DM mass $M_{\chi}=2.5 \mathrm{GeV}$. To get the observed dark matter abundance $\left(\sim 10^{-10}\right)$, we have used the branching ratio $B_{\chi}=2.2 \times 10^{-7}$. This shows that $\xi_{1}$ decay significantly to visible sector and rarely to invisible sector to get the correct relic abundance of dark matter and baryon asymmetry.

There are other possibile scattering processes which can populate the $\chi$ abundance are $\bar{\psi} \psi \rightarrow$ $\bar{\chi} \chi$ mediated by $Z_{D}$ and $\bar{f} f \rightarrow \bar{\chi} \chi$ through mixing of $Z$ and $Z_{D}$, in thermal bath apart from $\xi$ decay. In Fig. 2 we have shown the allowed parameter space, the blue region, in $g_{D}$ versus $M_{Z_{D}}$ plane where these processes remain in out-of-equilibrium and hence they remain consistent with relic abundance of DM obtained from $\xi$ decay.

\section{Direct detection of $\chi$ dark matter}

The spin independent elastic scattering cross-section of DM candidate with nuclei can be possible through the $Z-Z_{D}$ mixing. We get the total cross-section per nucleon to be,

$$
\sigma_{S I}^{Z} \simeq 2.171 \times 10^{-36} \mathrm{~cm}^{2} \tan ^{2} \theta_{Z} \frac{g_{D}^{2}}{\left(M_{Z_{D}} / \mathrm{GeV}\right)^{2}}
$$

where we have used DM mass to be $5 \mathrm{GeV}$. $\theta_{Z}$ is the mixing angle between $Z$ and $Z_{D}$. We can see the Fig. 2 where the spin independent direct DM detection cross-section allowed by the LUX [7] given by Red line corresponding to $5 \mathrm{GeV}$ DM. The values above the Red line are not allowed by the LUX limit.

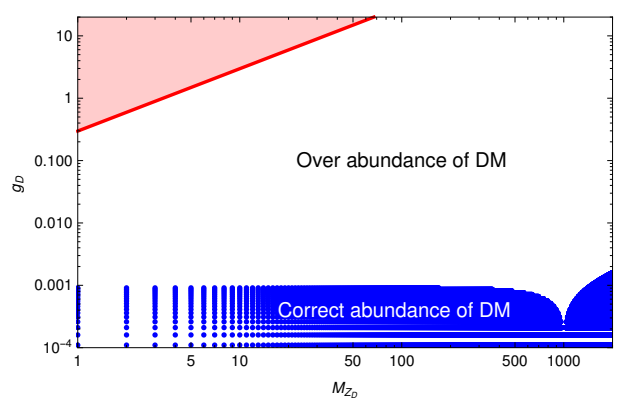

Figure 2: The LUX constraint on dark matter, arising via $Z-Z_{D}$ mixing, is shown on the plane of $g_{D}$ versus $M_{Z_{D}}$.

\section{References}

[1] M. Agostini et al. [GERDA Collaboration], Phys. Rev. Lett. 111, no. 12, 122503 (2013) [arXiv:1307.4720 [nucl-ex]].

[2] K. Dick, M. Lindner, M. Ratz and D. Wright, Phys. Rev. Lett. 84, 4039 (2000).

[3] H. Murayama and A. Pierce, Phys. Rev. Lett. 89, 271601 (2002) [hep-ph/0206177].

[4] D. G. Cerdeno, A. Dedes and T. E. J. Underwood, JHEP 0609, 067 (2006) [hep-ph/0607157].

[5] J. McDonald, N. Sahu and U. Sarkar, JCAP 0804, 037 (2008) [arXiv:0711.4820 [hep-ph]].

[6] N. Narendra, N. Sahoo and N. Sahu, Nucl. Phys. B 936, 76 (2018) [arXiv:1712.02960 [hep-ph]].

[7] D. S. Akerib et al. [LUX Collaboration], Phys. Rev. Lett. 118, no. 2, 021303 (2017) [arXiv:1608.07648 [astro-ph.CO]]. 